\title{
Peganum harmala Alkaloids Self-Assembled Supramolecular Nanocapsules with Enhanced Antioxidant and Cytotoxic Activities
}

Sherif Ashraf Fahmy ${ }^{1,2 \dagger}$, Marwa Y. Issa ${ }^{3}$, Basma M. Saleh ${ }^{1}$, Meselhy Ragab Meselhy ${ }^{3}$, Hassan Mohamed El-Said Azzazy*1

${ }^{1}$ Department of Chemistry, School of Sciences \& Engineering, The American University in Cairo, AUC Avenue, P.O. Box 74, New Cairo 11835, Egypt

${ }^{2}$ School of Life and Medical Sciences, University of Hertfordshire Hosted by Global Academic Foundation, R5 New Garden City, New Capital, Cairo, Egypt

${ }^{3}$ Department of Pharmacognosy, Faculty of Pharmacy, Cairo University, Kasr El-Aini Street, Cairo 11562, Egypt

$\dagger$ These authors contributed equally to this paper. 

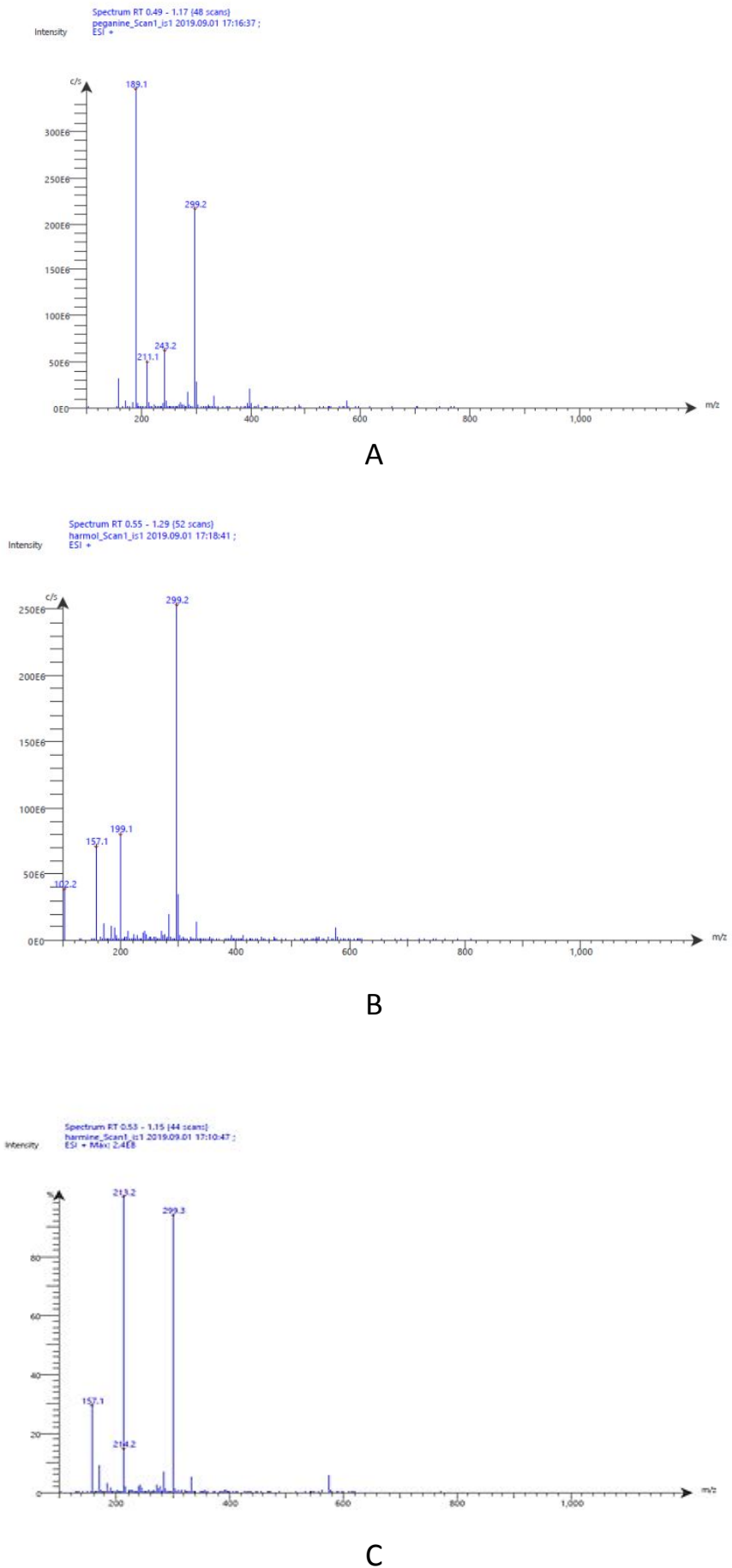

Figure S1: ESI/MS spectra at positive mode of Peganine (A), Harmol (B) and Harmine (C). 


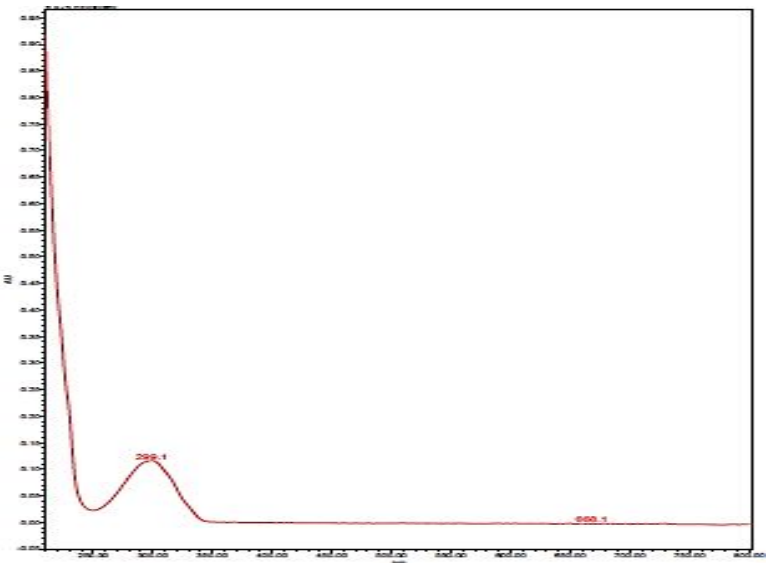

A

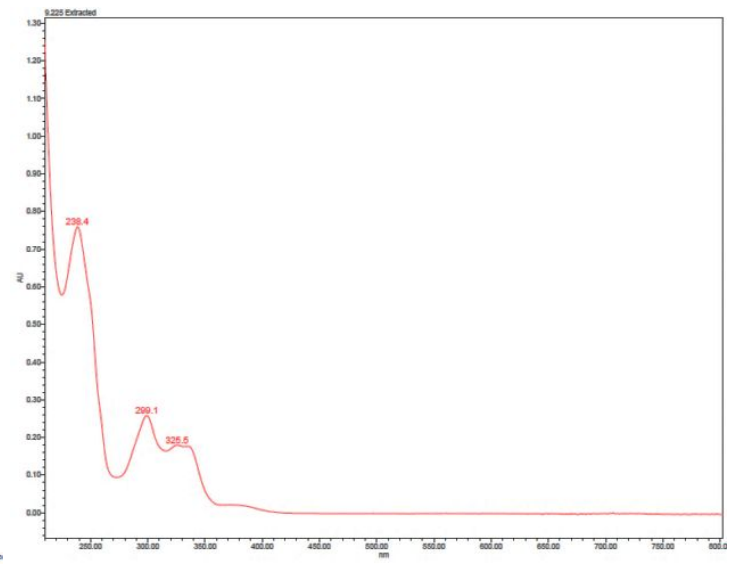

B

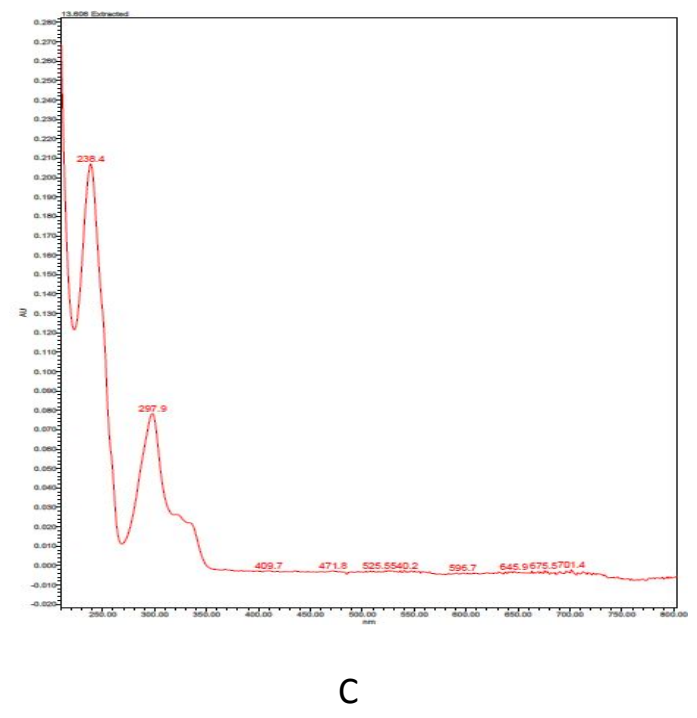

Figure S2: UV spectra of Peganine (A), Harmol (B) and Harmine (C). 


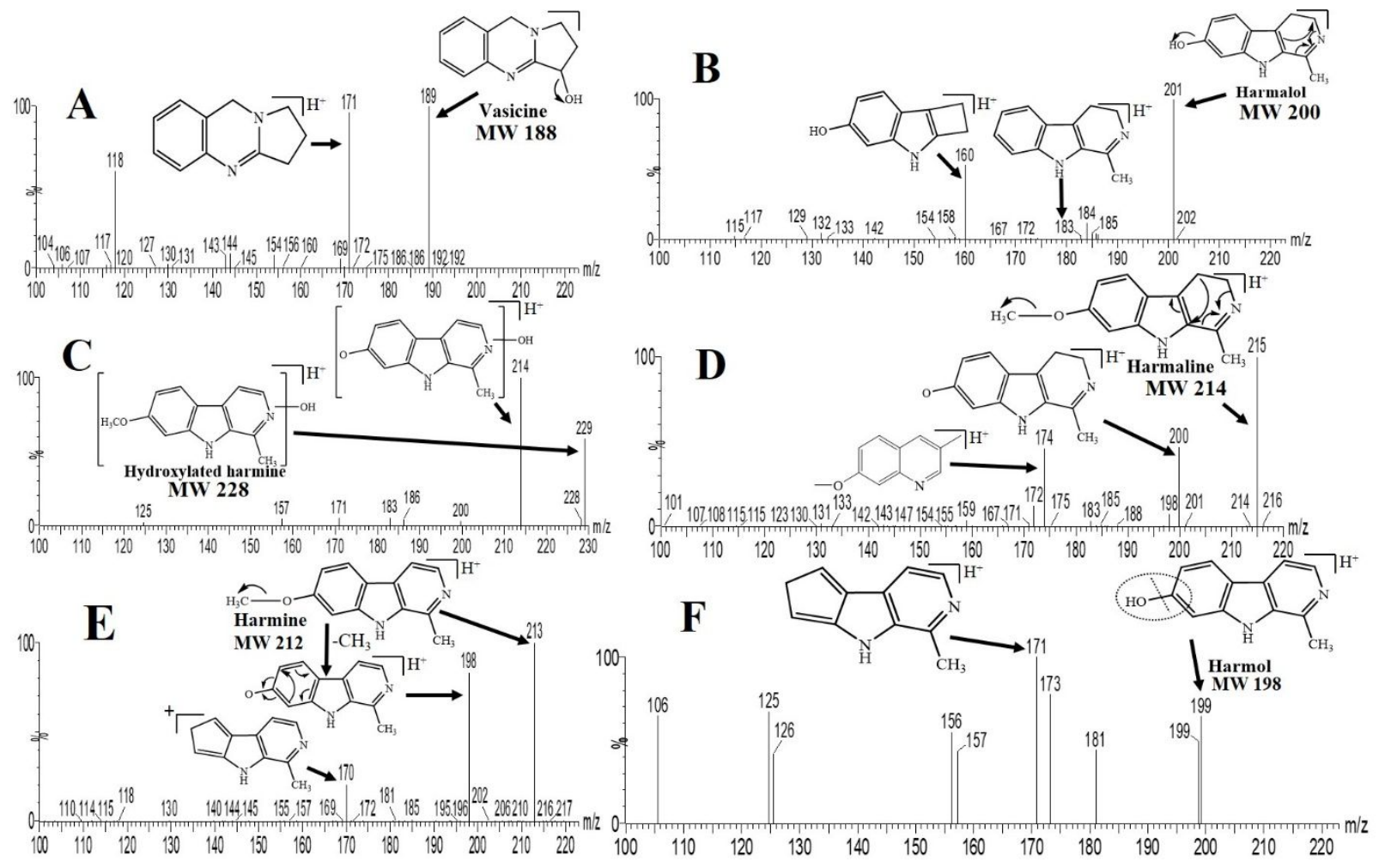

Figure S3. MS/MS fragmentation pattern of selected alkaloids; vasicine (A), harmalol (B), hydroxylated harmine (C), harmaline (D), harmine (E), and harmol (F), detected in harmala alkaloid-rich fraction by ultra-performance liquid chromatography-electrospray ionizationtandem mass spectrometry (UPLC/ESI-MS) using positive ion mode. 